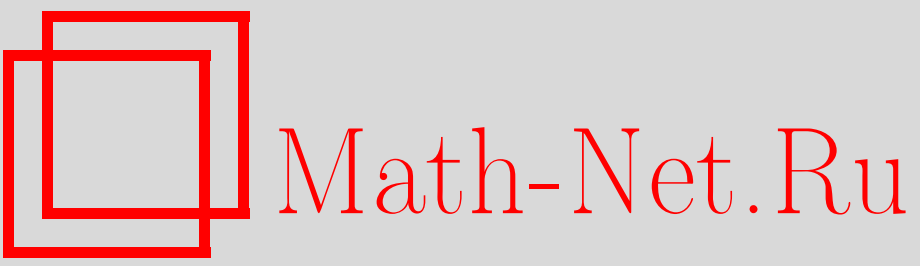

Л. В. Розовский, О малых уклонениях рядов независимых неотрицательных случайных величин с гладкими весами, Теория вероятн. и ее примен., 2013, том 58, выпуск 1, 133-151

DOI: https://doi.org/10.4213/tvp4497

Использование Общероссийского математического портала Math-Net.Ru подразумевает, что вы прочитали и согласны с пользовательским соглашением

http://www.mathnet.ru/rus/agreement

Параметры загрузки:

IP : 54.172 .240 .79

26 апреля 2023 г., $12: 38: 02$ 
ТЕОРИЯ ВЕРОЯТНОСТЕЙ

Том 58

И ЕЕ ПРИМЕНЕНИЯ

Выпуск 1

2013

(c) 2013 г.

РОзОВСКИЙ Л.В.*

\title{
О МАЛЫХ УКЛОНЕНИЯХ РЯДОВ НЕЗАВИСИМЫХ НЕОТРИЦАТЕЛЬНЫХ СЛУЧАЙНЫХ ВЕЛИЧИН С ГЛАДКИМИ ВЕСАМИ ${ }^{1)}$
}

\begin{abstract}
Изучаются логарифмические малые уклонения сумм $\sum_{j \geqslant 1} \lambda_{j} X_{j}$, где $\left\{X_{j}\right\}$ - независимые одинаково распределенные неотрицательные случайные величины и $\left\{\lambda_{j}\right\}$ - невозрастающая последовательность положительных чисел, удовлетворяющих некоторым условиям регулярности.

Ключевые слова и фразы: малые уклонения, неотрицательные случайные величины, медленно меняющиеся функции, правильно меняющиеся функции, преобразование Лапласа.
\end{abstract}

1. Введение. Пусть $S=\sum_{j \geqslant 1} \lambda_{j} X_{j}$, где $\left\{\lambda_{j}\right\}-$ последовательность невозрастающих положительных чисел, а $\left\{X_{i}\right\}$ - независимые копии неотрицательных случайных величин $X$ с функцией распределения $V(x)$, причем $\mathbf{P}(S<\infty)=1$. Нами исследуется поведение $-\ln \mathbf{P}(S<r)$ при $r \rightarrow 0$.

Аналогичные задачи рассматривались в значительном числе работ (подробные ссылки можно найти в [1]-[4]).

Отправной точкой для нашего исследования являются результаты из [1]-[3] и [5], [6]. В этих работах (см. также [9]) в предположении, что последовательность $\left\{\lambda_{j}\right\}$ удовлетворяет определенным условиям, были найдены асимптотики для $-\ln \mathbf{P}(S<r)$ при $r \rightarrow 0$ в явном виде при минимальных априорных предположениях. Приведем некоторые полученные там результаты, формулируя их, в основном, в терминах преобразований Лапласа случайных величин $X$ и $S$. По нашему мнению, это зачастую выглядит проще и короче. Равносильная переформулировка в терминах соответствующих распределений каких-либо затруднений не вызывает (см. п. 6).

Обозначим

$$
\varphi(t)=\mathbf{E} e^{-t X}, \quad \varphi_{S}(t)=\mathbf{E} e^{-t S}, \quad t \geqslant 0 .
$$

\footnotetext{
* Санкт-Петербургская химико-фармацевтическая академия, кафедра математики, Санкт-Петербург, Россия; e-mail: l_rozovsky@mail.ru

1) Работа выполнена при поддержке программы «Ведущие научные школы» (грант НШ-638.2008.1) и РФФИ (грант № 10-01-00242-а).
} 
Пусть положительная и невозрастающая функция $\lambda(u), u>0$, удовлетворяет условию $\lambda(j)=\lambda_{j}, j \geqslant 1$, а $\lambda^{-1}(\cdot)$ обозначает функцию, обратную к $\lambda(\cdot)$.

Теорема 1 [3, теорема 2.1]. Предположим, ито функиия $\lambda(u)$ правильно меняется на $\infty$ с некоторым показателем $-1 / \gamma<-1$ ( довательно, функиия $\lambda^{-1}(x)$ правильно меняется в нуле с показателем $\gamma \in(0,1))$. Пусть, кроме того, $-\ln \varphi(t)=O\left(t^{\gamma-\delta}\right), t \rightarrow \infty, u$ $\mathbf{E} X^{\gamma+\delta}<\infty$ при некотором $\delta>0$. Тогда $\mathbf{P}(S<\infty)=1 u$

$$
-\ln \varphi_{S}(t) \sim K(\gamma) \lambda^{-1}\left(\frac{1}{t}\right) \quad n p u t \rightarrow \infty
$$

где

$$
K(\gamma)=-\int_{0}^{\infty} u^{-\gamma} d \ln \varphi(u)<\infty
$$

Теорема 1 является обобщением одного результата работы [1], в которой соотношение (1.2) было получено в случае $\lambda_{j} \sim j^{-1 / \gamma} \ln ^{\delta} j$ при оптимальном моментном предположении.

Теорема 2 [3, теорема 4.1]. Пусть функиия $\lambda^{-1}(x)$ медленно меняется в нуле. Если $\mathbf{E} X^{\delta}<\infty$ при некотором $\delta>0 u$

$$
-(\ln \varphi(t))^{\prime} \sim \frac{\alpha}{t} \quad \text { npu } t \rightarrow \infty
$$

$m o \mathbf{P}(S<\infty)=1 u$

$$
-\ln \varphi_{S}(t) \sim \alpha \int_{1 / t}^{1} \lambda^{-1}(u) \frac{d u}{u} \quad n p u t \rightarrow \infty .
$$

Заметим, что соответствующий результат из [3], как нам кажется, содержит неточность (функция, определенная в [3, (7.4)], не обязательно медленно меняется), и теорема 2 представляет его исправленный вариант.

Нижеследующий результат является широким обобщением и уточнением теоремы 8 из [2].

Теорема 3 [6, теорема 5]. Пусть $-\ln \lambda_{j} \sim \alpha j$ при некотором $\alpha>0$ и $\mathbf{E} \ln (1+X)<\infty$. Если $-\ln \varphi(t)$ правильно меняется на $\infty$ с некоторым показателем $\delta \in(0,1)$, то $\mathbf{P}(S<\infty)=1, \Lambda_{\delta}=\sum_{j \geqslant 1} \lambda_{j}^{\delta}<\infty u$ $\ln \varphi_{S}(t) \sim \Lambda_{\delta} \ln \varphi(t)$ nри $t \rightarrow \infty$.

Основной целью настоящей работы является получение новых асимптотик для $-\ln \varphi_{S}(t)$ при $t \rightarrow \infty$, а также уточнение и обобщение утверждений типа теорем 1-3. В частности, удается несколько ослабить предположения относительно весов $\left\{\lambda_{j}\right\}$, а также получить асимптотики, такие как (1.2) и (1.5), при менее ограничительных, зачастую неулучшае- 
мых, условиях, в том числе в ситуациях, когда сформулировать результат в терминах распределений без дополнительных предположений не представляется возможным.

Несколько слов о структуре статьи. Работа разбита на шесть пунктов, причем п. 5 содержит доказательства, а в справочном п. 6 приведены тауберовы теоремы, устанавливающие связь между распределением случайной величины и ее преобразованием Лапласа. В пп. 2 и 3 предлагаются условия, при которых $-\ln \varphi_{S}(t)$ имеет асимптотику, правильно меняющуюся на $\infty$ и не зависящую от любого конечного подмножества весов $\left\{\lambda_{j}\right\}$.

Теорема 1 является следствием основного результата п. 2, а именно п. (1) теоремы 4, в условиях которого имеет место соотношение (1.3). В замечании 1 показано, как утверждение (1) теоремы 4 выглядит в терминах распределений. В следствиях 1, 2 и 3, также сформулированных в терминах распределений, приведены необходимые и достаточные условия, при которых $-\ln \mathbf{P}(S<r), r \rightarrow 0$, имеет соответствующую асимптотику для случая весов $\left\{\lambda_{j}\right\}$ некоторого определенного вида.

В п. 3 исследуется ситуация $K(\gamma)=\infty$ (см. (1.3)), при которой часть (2) теоремы 4, а также следствия 1 и 2 могут быть уточнены. Приведены теоремы 5 и 6 (интеграл $K(\gamma)$ расходится на $\infty$ ) и теорема 7 $(K(\gamma)$ расходится в нуле), содержащие разнообразные условия, при которых асимптотику для $-\ln \varphi_{S}(t)$ удается выписать в явном виде. В частности, показано, что теорема 2 вытекает из теоремы 6 .

В п. 4 приведены теоремы 8, 9 и 10, в которых изучается случай, когда асимптотика $-\ln \varphi_{S}(t)$, в отличие от результатов пп. 2 и 3 , определенным образом зависит от значений $\lambda_{1}, \lambda_{2}, \ldots$. При этом теорема 8 из п. 4 является широким обобщением теоремы 3 , а асимптотика, найденная в теореме 10, является новой и ранее в литературе не встречалась.

Следует сказать, что в доказательствах ключевую роль играет предложение 1 из [6]. Мы также пользуемся некоторыми соображениями из [5].

2. Результаты. I. Пусть положительная неубывающая функция $b(u)=b_{\gamma}(u), u \geqslant 0$, правильно меняется на $\infty$ с некоторым показателем $\gamma, 0 \leqslant \gamma \leqslant 1$, т.е. функция $b_{0}(u)=u^{-\gamma} b(u)$ медленно меняется на $\infty$. Следуя [5] и [6], предположим, что $b(u)$ и $\left\{\lambda_{j}\right\}$ связаны соотношением

$$
b_{\gamma}\left(\frac{1}{\lambda_{j}}\right) \sim j \quad \text { при } j \rightarrow \infty \text {. }
$$

Так, если $\lambda_{j} \sim j^{-1 / \gamma} g(\ln j)$, где $\gamma>0$ и положительная функция $g(u)$, $u>0$, при некотором $\delta$ удовлетворяет условию $u g^{\prime}(u)=(\delta+o(1)) g(u)$, $u \rightarrow \infty$ (и, следовательно, правильно меняется на $\infty$ с показателем $\delta$ ), то можно положить $b(u)=u^{\gamma}\left(\gamma^{\delta} g(\ln u)\right)^{\gamma}, u>1$. Если же $-\ln \lambda_{j} \sim \alpha j^{\delta}$ 
при положительных $\alpha$ и $\delta$, то $b(u)=\left(\alpha^{-1} \ln u\right)^{1 / \delta}, u>1$. Наконец, если $\lambda^{-1}(1 / u)$ правильно меняется на $\infty$ с положительным показателем $\gamma$, то естественен выбор $b(u)=\lambda^{-1}(1 / u), u>1$.

В [5, замечание 1] было показано, что необходимое предположение $\mathbf{P}(S<\infty)=1$ равносильно условию

$$
\int_{1}^{\infty}\left(u \int_{u}^{\infty} b(y) \frac{d y}{y^{2}}\right) d V(u)<\infty
$$

которое при $0 \leqslant \gamma<1$ совпадает с $\mathbf{E} b_{\gamma}(X)<\infty$.

Введем дополнительные обозначения. Предполагая, что выполнено (2.1), положим

$$
\begin{gathered}
g(u)=g_{\gamma}(u)=\sup _{t \geqslant 1} \frac{b(u t)}{b(t)}, \\
h(u)=h_{\gamma}(u)=\sup _{t \geqslant 1} \frac{b(t)}{b(u t)}, \quad \tilde{h}(u)=\tilde{h}_{\gamma}(u)=\sup _{t \geqslant 1} \frac{b(u t)}{b\left(u^{2} t\right)} .
\end{gathered}
$$

Перечислим некоторые свойства введенных выше функций.

Лемма 1. (1) Функиия $g(u)$ не убывает и правильно меняется на $\infty$ с показателем $\gamma$. Кроме того, $g(u) \geqslant u^{\gamma}$.

(2) Функиии $h(u)$ и $\tilde{h}(u)$ не возрастают и правильно меняются на $\infty$ с показателем - $\gamma$. Кроме того,

$$
\begin{gathered}
\tilde{h}(u) \leqslant h(u), \quad \frac{1}{h(u)} \leqslant \frac{b(u)}{b(1)} \leqslant g(u), \\
h(u) \geqslant u^{-\gamma}, \quad \tilde{h}(u) \geqslant u^{-\gamma}, \quad \tilde{h}(u) \geqslant \frac{b(u)}{b\left(u^{2}\right)} .
\end{gathered}
$$

(3) Предположим, что положительная функиия $L(u)$ удовлетворяет условию

$$
u(\ln L(u))^{\prime} \searrow 0 \quad n p u u \rightarrow \infty
$$

( $u$, следовательно, $L(u)$ не убьввает и медленно меняется на $\infty$.)

Ecлu $b(u)=u^{\gamma} L(u), \operatorname{mo} h(u)=\tilde{h}(u)=u^{-\gamma} u$

$$
g(u)=O(b(u)), \quad u \rightarrow \infty
$$

если $b(u)=u^{\gamma} / L(u), \operatorname{mog} g(u)=u^{\gamma} u$

$$
\begin{array}{ll}
h(u)=O\left(\frac{1}{b(u)}\right), & u \rightarrow \infty, \\
\tilde{h}(u)=O\left(\frac{b(u)}{b\left(u^{2}\right)}\right), & u \rightarrow \infty .
\end{array}
$$


Соотношение (2.5) было доказано в [5, замечание 2]; соотношения (2.6) и (2.7) проверяются аналогично. Все эти оценки неулучшаемы.

Заметим, что если наряду с (2.4) выполнено условие $u \ln u(\ln L(u))^{\prime}=$ $O(1)$, то $1 \leqslant u^{\gamma} \tilde{h}(u)=O(1)$.

Пусть, так же как в теореме $1, K(\gamma)=-\int_{0}^{\infty} u^{-\gamma} d \ln \varphi(u), \gamma \geqslant 0$.

Нетрудно проверить, что $K(0)=-\ln \mathbf{P}(X=0), K(1)=\infty$ и $K(\gamma)=-\gamma \int_{0}^{\infty} \ln \varphi(u) u^{-\gamma} d u / u$ при $0<\gamma<1$. Кроме того, интеграл $K(\gamma)$ сходится в нуле тогда и только тогда, когда $\mathbf{E} X^{\gamma}<\infty$.

Теперь сформулируем основной результат этого пункта.

Теорема 4. (1) Пусть (см. (2.1)) $0 \leqslant \gamma<1 u$

$$
\mathbf{E} g_{\gamma}(X)<\infty
$$

Предположим, что либо

$$
-\int_{1}^{\infty} h(u) d \ln \varphi(u)<\infty,
$$

либо, если $0<\gamma<1$, найдется функиия $\kappa(u)$,

$$
\kappa(u) \searrow 0 \quad \text { npu } u \rightarrow \infty
$$

такая, что

$$
-\ln \varphi(t)=O(\kappa(t) b(t)) \quad \text { nрu } t \rightarrow \infty
$$

$u$

$$
\int_{1}^{\infty} \kappa(u) b(u) \tilde{h}(u) \frac{d u}{u}<\infty .
$$

Тогда $\mathbf{P}(S<\infty)=1, K(\gamma)<\infty u$

$$
\lim _{t \rightarrow \infty} \frac{-\ln \varphi_{S}(t)}{b(t)}=K(\gamma) .
$$

(2) Пусть (см. (2.1)) $0 \leqslant \gamma \leqslant 1$. Если $\mathbf{P}(S<\infty)=1$ и $K(\gamma)=\infty$, то соотношение (2.13) остается справедливым.

Заметим (см. лемму 1), что из (2.8) следует $\mathbf{E} X^{\gamma}<\infty$, а также то, что (2.9) при $\gamma>0$ равносильно $-\int_{1}^{\infty} \ln \varphi(u) h(u) d u / u<\infty$, причем последнее предположение, вообще говоря, ограничительнее, нежели (2.12).

3 а м е ч а н и е 1 . В соответствии с леммой 5 (см. п. 6), соотношение (2.13) равносильно

$$
\lim _{r \rightarrow 0} \frac{-\ln \mathbf{P}(S<r)}{b(s)}=C(\gamma)
$$

здесь $s=s(r) \rightarrow \infty$ и выполнено условие $b(s) / s \sim r, r \rightarrow 0$, а $C(\gamma)=$ $c_{*}(\gamma)(K(\gamma))^{1 /(1-\gamma)}$, где $c_{*}(\gamma)=(1-\gamma) \gamma^{\gamma /(1-\gamma)}\left(c_{*}(0)=\lim _{\gamma \rightarrow 0} c_{*}(\gamma)=1\right)$. 
Условие (2.11) также может быть переписано в терминах $-\ln V(r)$ (по крайней мере, для случая правильно меняющейся функции $\kappa(t))$.

Из первого утверждения леммы 1 следует, что (2.8) вытекает из $\mathbf{E} X^{\gamma+\delta}<\infty$ при некотором $\delta>0$, а ее второе утверждение, в свою очередь, показывает, что сделанное в теореме 1 предположение

$$
-\ln \varphi(t)=O\left(t^{\gamma-\delta}\right), \quad t \rightarrow \infty,
$$

влечет (2.11) и (2.12). Таким образом, теорема 1 является следствием теоремы 4.

Другое следствие теоремы 4 , относящееся к случаю $\gamma=0$, ведет к [5, предложение 1].

3 а м е ч а н и е 2. Пусть выполнено (2.4). Если $b(u)=u^{\gamma} L(u)$, то (2.8) превращается в необходимое условие

$$
\mathbf{E} b(X)<\infty,
$$

а условия (2.9) и (2.12) совпадают с условиями

$$
-\int_{1}^{\infty} u^{-\gamma} d \ln \varphi(u)<\infty
$$

и $\int_{1}^{\infty} \kappa(u) L(u) d u / u<\infty$ соответственно. Если $b(u)=u^{\gamma} / L(u)$, то $(2.8)$ совпадает с $\mathbf{E} X^{\gamma}<\infty$, а условия (2.9), (2.12) переходят соответственно в условия $-\int_{1}^{\infty} 1 / b(u) d \ln \varphi(u)<\infty$ и

$$
\int_{1}^{\infty} \kappa(u) \frac{L\left(u^{2}\right)}{L^{2}(u)} \frac{d u}{u}<\infty
$$

В частности, условия (2.11) и (2.17) выполняются при любом $\kappa(u) \searrow 0$, если

$$
\int_{1}^{\infty} \frac{L\left(u^{2}\right)}{L^{2}(u)} \frac{d u}{u}<\infty
$$

и

$$
-\ln \varphi(u)=o(b(u)) \quad \text { при } u \rightarrow \infty .
$$

Заметим, что в соответствии с леммой 5 из п. 6 и предложением 2 из [6] условие (2.19) равносильно $-\ln V(r)=o(b(s)), r \rightarrow 0$, и в некотором смысле является необходимым для (2.13) (см. лемму 2 из п. 5 и высказывание сразу после нее).

Следствие 1. Пусть выполнено условие (2.4) и $b(u)=u^{\gamma} L(u) n p u$ $0 \leqslant \gamma<1$. Тогда соотношения (2.15) $u$

$$
C(\gamma)=A \in[0, \infty]
$$


являются необходимыми и достаточными для выполнения соотношений $\mathbf{P}(S<\infty)=1$ u

$$
\lim _{r \rightarrow 0} \frac{-\ln \mathbf{P}(S<r)}{b(s)}=A
$$

(см. обозначения в (2.14)).

Следствие 2. Пусть вылолнень условия (2.4) $и b(u)=u^{\gamma} / L(u)$ при $0<\gamma<1$. Пусть также справедливь предположения (2.10), (2.11) и (2.17). Тогда условия (2.15) и (2.20) являются необходимьими и достаточнылми для $\mathbf{P}(S<\infty)=1 u(2.21)$.

Следствие 3. Пусть $\lambda_{j} \sim\left(j \ln ^{\delta} j\right)^{-1 / \gamma}$ при $1 / \gamma>1$ и некотором $\delta$. Обозначим $\alpha=\gamma /(1-\gamma)$ и дополнительно предположим, что при $\delta>1$

$$
-\ln V(r)=o\left(r^{-\alpha} \ln ^{-\delta /(1-\gamma)} \frac{1}{r}\right), \quad r \rightarrow 0,
$$

а если $0<\delta \leqslant 1$, mo

$$
-\ln V(r)=O\left(r^{-\alpha} \ln ^{-\rho} \frac{1}{r}\right), \quad r \rightarrow 0,
$$

при некотором $\rho>(1-\gamma)^{-1}$. Условия $\mathbf{P}(S<\infty)=1$ и

$$
\lim _{r \rightarrow 0}\left(r^{\gamma} \ln ^{\delta} \frac{1}{r}\right)^{1 /(1-\gamma)}(-\ln \mathbf{P}(S<r))=A \in[0, \infty]
$$

виполняются тогда и только тогда, когда $\mathbf{E} X^{\gamma} \ln ^{-\delta} X<\infty u K(\gamma)=$ $A^{1-\gamma}(1-\gamma)^{-1} \alpha^{\delta-\gamma}$ (таким образом, в $\left.(2.22) A=\left[\alpha^{\gamma-\delta}(1-\gamma) K(\gamma)\right]^{1 /(1-\gamma)}\right)$.

Следствие 3 , в частности, уточняет соответствующий результат из [1], упомянутый в п. 1.

3. Результаты. II. В этом пункте рассматривается ситуация $K(\gamma)=\infty$, при которой вторая часть теоремы 4, а также следствия 1 и 2 могут быть уточнены.

Теорема 5. Пусть функиия $l_{\gamma}(t)=-\int_{1}^{t} \ln \varphi(u) u^{-1-\gamma} d u, t \geqslant 1, n p u$ некотором $\gamma, 0 \leqslant \gamma<1$, медленно меняется на $\infty$ или, равносильно,

$$
-\ln \varphi(u)=o(1) u^{\gamma} l_{\gamma}(u), \quad u \rightarrow \infty,
$$

u пусть, кроме того, $l_{\gamma}(\infty)=\infty$.

Предположим также, что в случае $\gamma=0$

$$
\mathbf{E} \ln (1+X)<\infty \quad u \quad \ln \lambda_{j} \sim-\alpha j(\exists \alpha>0),
$$

а в случае $0<\gamma<1$

$$
\mathbf{E} X^{\gamma}<\infty \quad u \quad \lambda_{j} \sim j^{-1 / \gamma}
$$


Тогда $\mathbf{P}(S<\infty)=1$ u

$$
-\ln \varphi_{S}(t) \sim c(\gamma) t^{\gamma} l_{\gamma}(t), \quad t \rightarrow \infty,
$$

где $c(0)=1 / \alpha$ и $c(\gamma)=\gamma(0<\gamma<1)$.

Напомним, что моментные условия в (3.2) и (3.3) являются необходимыми для $\mathbf{P}(S<\infty)=1$.

Следствие 4. Пусть $\delta>-1$ u $\beta \geqslant 0$ - некоторые постоянные, а функиия $H(u)$ правильно меняется на $\infty$ с показателем $\delta(1+\beta), u$ nусть

$$
-\ln V(r) \sim\left(\frac{1}{r}\right)^{\beta} H\left(\ln \frac{1}{r}\right), \quad r \rightarrow 0 .
$$

Если при $\beta=0$ выполнено условие (3.2), а при $\beta>0-$ условие (3.3), в котором $\gamma=\beta /(1+\beta)$, то $\mathbf{P}(S<\infty)=1 u$

$$
-\ln \mathbf{P}(S<r) \sim A(\beta)(-\ln V(r))\left(\ln \frac{1}{r}\right)^{1+\beta}, \quad r \rightarrow 0,
$$

где $A(0)=(\alpha(1+\delta))^{-1}$ и $A(\beta)=(\beta /(1+\delta))^{1+\beta}$, если $\beta>0$.

Следствие 4 можно вывести из теоремы 5 , воспользовавшись леммой 5 и нижеследующим замечанием 3.

3 а м е ч а н и е 3 . Пусть $-\ln \varphi(u) \sim u^{\gamma} h(\ln u), u \rightarrow \infty$, где $h(t)$ правильно меняется на $\infty$ с некоторым показателем $\delta>-1$. Тогда

$$
l_{\gamma}(t) \sim(1+\delta)^{-1} h(\ln t) \ln t, \quad t \rightarrow \infty .
$$

Отметим, что при $\gamma=0$ показатель $\delta$ должен быть неотрицательным, а функция $h(t)$ может быть положительной постоянной.

Если в следствии 4 положить $\beta=0$ и $H(u)=c$ или $c u$, где $c-$ положительная постоянная, получим уточненные варианты теорем 2 и 4 из $[2]$.

Теорема 6. Пусть при некотором $\gamma, 0 \leqslant \gamma<1$, выполняются условия (2.1) и (2.8). При $t \geqslant 1$ положим $l_{\gamma}(t)=\int_{1}^{t} b_{\gamma}(u) u^{-1-\gamma} d u$, предполагая, ито $l_{\gamma}(\infty)=\infty$. Пусть в случае $\gamma=0$

$$
-\ln \varphi(u) \sim c \ln u, \quad u \rightarrow \infty,
$$

а в случае $0<\gamma<1$

$$
-\ln \varphi(u) \sim c u^{\gamma}, \quad u \rightarrow \infty,
$$

при некоторой положительной постоянной $c$.

Тогда выполняются условия $\mathbf{P}(S<\infty)=1 u(3.4)$, где $c(0)=c u$ $c(\gamma)=c \gamma(0<\gamma<1)$. 
Напомним (см. замечание 1 или лемму 5), что условия (3.6) и (3.7) равносильны соответственно условиям $-\ln V(r) \sim c \ln (1 / r)$ и $-\ln V(r) \sim$ $c_{*}(\gamma) c^{1 /(1-\gamma)}(1 / r)^{\gamma /(1-\gamma)}$ при $r \rightarrow 0$.

Отметим, что (3.6) влечет (1.4) и, следовательно (см. также лемму $1(1))$, теорема 2 следует из теоремы 6 .

3 а м е ч а н и е 4 . Пусть $b_{\gamma}(u) \sim u^{\gamma} h(\ln u), u \rightarrow \infty$, где функция $h(t)$ определена в замечании 3 . Тогда имеет место (3.5).

Отсюда, в частности, следует, что теорема 6 при $b_{\gamma}(u)=u^{\gamma}(\gamma \ln u)^{\delta}$ приводит к теореме $5(1)$ из [6].

В теоремах 5 и 6 интеграл $K(\gamma)$ расходился на $\infty$. Теперь рассмотрим случай, когда $K(\gamma)$ расходится в нуле.

Теорема 7. Пусть при некотором $\gamma, 0<\gamma<1$, выполняется условие (2.1). Для $t \geqslant 1$ положим $l_{\gamma}(t)=\int_{t}^{\infty} b_{\gamma}(u) u^{-1-\gamma} d u<\infty$. Если при некотором положительном с

$$
1-\varphi(u) \sim c u^{\gamma}, \quad u \rightarrow 0,
$$

$u$

$$
-\ln \varphi(t)=O\left(t^{\delta}\right), \quad t \rightarrow \infty,
$$

где $\delta<\gamma$, то выполняются условия $\mathbf{P}(S<\infty)=1$ и (3.4) при $c(\gamma)=c \gamma$.

Заметим, что (3.8) равносильно условию $1-V(x) \sim c(\Gamma(1-\gamma))^{-1} x^{-\gamma}$, $x \rightarrow \infty$, а (3.9) равносильно условию $-\ln V(r)=O\left(r^{-1 /(1-\delta)}\right), r \rightarrow 0$.

3 а м е ч а н и е 5 . Функция $l_{\gamma}(t)$ медленно меняется на $\infty$. В частности, если $b_{\gamma}(u) \sim u^{\gamma} h(\ln u), u \rightarrow \infty$, где функция $h(t)$ правильно меняется на $\infty$ с некоторым показателем $\delta<-1$, то, аналогично (3.5), $l_{\gamma}(t) \sim|1+\delta|^{-1} h(\ln t) \ln t, t \rightarrow \infty$.

Простое достаточное условие (3.9) в некоторых случаях может быть ослаблено вплоть до оптимального предположения (3.1).

Положим $L(u)=u^{-\gamma} b_{\gamma}(u), \tilde{l}_{\gamma}(u)=\sup _{t \geqslant 1} l_{\gamma}(u t) / l_{\gamma}\left(u^{2} t\right)$, а также $\mu(u)=L(u) / l_{\gamma}(u)$ и $\bar{\mu}(u)=\sup _{t \geqslant 1} \mu(u t) / \mu(u)$.

Функции $L(u), \tilde{l}_{\gamma}(u)$ и $\bar{\mu}(u)$ медленно меняются на $\infty$. Помимо этого, $\tilde{l}_{\gamma}(u) \geqslant 1, \lim _{u \rightarrow \infty} \mu(u)=0$ и $\bar{\mu}(u) \geqslant 1$.

3 а м е ч а н и е 6. (1) Если

$$
\int_{1}^{\infty} \tilde{l}_{\gamma}(u) L(u) \frac{d u}{u}<\infty,
$$

то условие (3.9) в теореме 7 можно заменить на (3.1).

(2) Пусть $\kappa(u) \searrow 0$ при $u \rightarrow \infty$. Если

$$
\int_{1}^{\infty} \kappa(u) \bar{\mu}(u) \tilde{l}_{\gamma}(u) L(u) \frac{d u}{u}<\infty,
$$

то условие (3.9) в теореме 7 можно заменить на

$$
-\ln \varphi(t)=O\left(\kappa(t) t^{\gamma} l_{\gamma}(t)\right), \quad t \rightarrow \infty .
$$


Выбирая $\kappa(u)=t^{-\varepsilon}$ при некотором достаточно малом $\varepsilon>0$, видим, что условие (3.11) выполняется, а (3.9) вытекает из (3.12).

Отметим, что если $\mu(u) \searrow 0$, то $\tilde{l}_{\gamma}(u)=l_{\gamma}(u) / l_{\gamma}\left(u^{2}\right)$ и $\bar{\mu}(u)=1$. Если дополнительно предположить, что $\mu(u)=O(1 / \ln u)$ при $u \rightarrow \infty$, то $1 \leqslant \tilde{l}_{\gamma}(u)=O(1)$ и интеграл в $(3.10)$ сходится.

Одно следствие теоремы 7 , леммы 5 и замечаний 5 и 6 (1) приведено в следующем пункте (см. следствие 5 при $K=0)$.

4. Результаты. III. В этом пункте мы предполагаем, что имеет место (2.1) и

$$
-\ln \varphi(t) \sim \omega_{\delta}(t) \quad \text { при } t \rightarrow \infty,
$$

где $\omega_{\delta}(t), t \geqslant 1$, является положительной функцией, правильно меняющейся на $\infty$ с некоторым показателем $\delta, 0<\delta \leqslant 1$.

Теорема 8. Пусть $0 \leqslant \gamma<\delta$. Если (см. (2.1)) $\mathbf{E} b_{\gamma}(X)<\infty$, mo

$$
\ln \varphi_{S}(t) \sim \Lambda_{\delta} \ln \varphi(t) \quad \text { npu } t \rightarrow \infty,
$$

где

$$
\Lambda_{\delta}=\sum_{j \geqslant 1} \lambda_{j}^{\delta}<\infty .
$$

Отметим, что теорема 3 является следствием теоремы 8 при $\delta \in$ $(0,1), \gamma=0$ и $b_{\gamma}(u)=\alpha^{-1} \ln u, u>1$.

3 а м е ч а н и е 7. По лемме 5 условия (4.1) и (4.2) при $\delta<1$ равносильны соответственно условию правильного изменения функции $-\ln V(r)$ в нуле с показателем $\delta /(1-\delta)$ и условию $\ln \mathbf{P}(S<r) \sim$ $\Lambda_{\delta}^{1 /(1-\delta)} \ln V(r), r \rightarrow 0$.

Далее рассмотрим граничную ситуацию, когда параметр $\delta$ из условия (4.1) совпадает с $\gamma$ из условия (2.1).

Для того чтобы сформулировать результаты в оптимальной общности, нам понадобятся дополнительные обозначения:

$$
\begin{gathered}
\omega(u)=u^{-\gamma} \omega_{\gamma}(u), \quad \bar{\omega}(u)=\sup _{t \geqslant 1} \frac{\omega(u t)}{\omega(t)}, \quad \widetilde{\omega}(u)=\sup _{t \geqslant 1} \frac{\omega(u t)}{\omega\left(u^{2} t\right)}, \\
\nu(u)=\frac{b_{\gamma}(u)}{\omega_{\gamma}(u)}, \quad \bar{\nu}(u)=\sup _{t \geqslant 1} \nu(u t), \quad u \geqslant 1 .
\end{gathered}
$$

Заметим, что все определенные выше функции медленно меняются на $\infty$, причем $\bar{\omega}(u) \geqslant 1$ и $\widetilde{\omega}(u) \geqslant 1$. Кроме того, функция $\bar{\nu}(u)$ не возрастает и, следовательно, имеет неотрицательный предел $\bar{\nu}(\infty)$ на $\infty$.

Теорема 9. Пусть $0<\delta=\gamma<1$. Предположим, что выполнены условия (4.1) (с $\delta=\gamma)$,

$$
\int_{1}^{\infty} \bar{\nu}(u) \widetilde{\omega}(u) \omega(u) \frac{d u}{u}<\infty
$$


$u$

$$
\mathbf{E} \bar{\nu}(X) \bar{\omega}(X) X^{\gamma}<\infty .
$$

Тогда $\mathbf{P}(S<\infty)=1, \Lambda_{\gamma}<\infty$ (см. (4.3)) u

(1) если $\bar{\nu}(\infty)=0$, то имеет место (4.2) при $\delta=\gamma$;

(2) если $\bar{\nu}(\infty)>0$, то $K(\gamma)<\infty($ см. (1.2)) u

$$
-\ln \varphi_{S}(t) \sim \Lambda_{\gamma} \omega_{\gamma}(t)+K(\gamma) b_{\gamma}(t) \quad \text { npu } t \rightarrow \infty .
$$

Обращаем внимание на то, что при $\bar{\nu}(\infty)>0$ величины $\bar{\nu}(\cdot)$ из условий (4.5) и (4.6) можно удалить.

3 а м е ч а н и е 8 . (1) Если

$$
u(\ln \omega(u))^{\prime} \searrow 0 \quad \text { при } u \rightarrow \infty,
$$

то $\bar{\omega}(u)=O(\omega(u))$ и $\widetilde{\omega}(u)=O(1)$ при $u \rightarrow \infty$, а если

$$
u(\ln \omega(u))^{\prime} \nearrow 0 \quad \text { при } u \rightarrow \infty,
$$

то $\bar{\omega}(u)=O(1)$ и $\widetilde{\omega}(u)=O\left(\omega(u) / \omega\left(u^{2}\right)\right)$ при $u \rightarrow \infty$, причем и в том, и в другом случае полученные оценки являются неулучшаемыми.

(2) Пусть $\nu(u)$, начиная с некоторого места, не возрастает. Тогда из (4.7) следует $\bar{\nu}(\infty)=0$, а условия (4.5) и (4.6) равносильны «необходимым» предположениям $l_{\gamma}(1)<\infty$ и $(2.15)$ соответственно (см. [6, предложение 3] и обозначения в теореме 7); если же имеет место (4.8), то (4.5) равносильно условию $\int_{1}^{\infty} b_{\gamma}(u) u^{-(1+\gamma)} \omega(u) / \omega\left(u^{2}\right) d u<\infty$, а (4.6) - условию $\mathbf{E} b_{\gamma}(X) / \omega(X)<\infty$.

Отметим, что если (наряду с (4.8))

$$
u(\ln u)\left(\ln \frac{1}{\omega(u)}\right)^{\prime}=O(1), \quad u \rightarrow \infty
$$

то $1 \leqslant \omega(u) / \omega\left(u^{2}\right)=O(1), u \rightarrow \infty$, и, следовательно, условие (4.5) опять является необходимым.

Используя замечание 8, из теоремы 9 можно вывести ряд более прозрачных следствий, таких, например, как теорема 6 работы [6], где был проанализирован частный случай $\omega_{\gamma}(t)=c t^{\gamma} \ln ^{\delta} t, b_{\gamma}(t)=t^{\gamma}(\gamma \ln t)^{\delta}$.

Следующее ниже утверждение, ранее не встречавшееся в литературе, использует обозначения из теоремы 7 и замечания 6 .

Теорема 10. Пусть (см. (2.1) и (4.1)) $\delta=\gamma$ и выполнень условия (3.8) и (4.5). Тогда $\mathbf{P}(S<\infty)=1, \Lambda_{\gamma}<\infty u$

$$
-\ln \varphi_{S}(t) \sim \Lambda_{\gamma} \omega_{\gamma}(t)+c \gamma t^{\gamma} l_{\gamma}(t), \quad t \rightarrow \infty .
$$


Следствие 5. Пусть выполнены следующие условия:

(1) $\lambda_{j} \sim\left(j g_{\delta}(\ln j)\right)^{-1 / \gamma}$, где Функиия $g_{\delta}(u)$ правильно меняется на $\infty$ с некоторым показателем $\delta>1$;

(2) $1-V(x) \sim a x^{-\gamma}, x \rightarrow \infty$;

(3) $-\ln V(r)=(K+o(1)) \tau(r), r \rightarrow 0$, при некотором $K \geqslant 0$, где

$$
\tau(r)=\left(\frac{1}{r}\right)^{\alpha}\left(\frac{\ln (1 / r)}{g_{\delta}(\ln (1 / r))}\right)^{1 /(1-\gamma)} \quad u \quad \alpha=\frac{\gamma}{1-\gamma} .
$$

Тогда $\mathbf{P}(S<\infty)=1, \Lambda_{\gamma}<\infty u$

$$
-\ln \mathbf{P}(S<r) \sim A \tau(r), \quad r \rightarrow 0,
$$

где $A=\left[K^{1-\gamma} \Lambda_{\gamma}+\alpha^{\gamma-\delta} \gamma(1-\gamma) a \Gamma(1-\gamma) /(\delta-1)\right]^{1 /(1-\gamma)}$.

Следствие 5 при $K=0$ обобщает одно утверждение из [6, теорема 4] и, как уже говорилось ранее, вытекает из теоремы 7. В случае положительного $K$ для проверки используются теорема 10, лемма 5 и замечания $5,6(1)$ и 8.

5. Доказательства. Положим (см. (1.1) и (2.1))

$$
J(t)=J_{\gamma}(t)=-\int_{1}^{\infty} \ln \varphi\left(\frac{t}{u}\right) d b_{\gamma}(u), \quad t \geqslant 0 .
$$

Лемма 2 [6, предложение 1]. Пусть $\omega(t)$ обозначает положительную функцию, которая правильно меняется на $\infty$ с некоторым показателем $\tau \in[0,1]$. Предположим, что выполнены условия (2.1) и (2.2) (или $\mathbf{P}(S<\infty)=1)$.

(1) Eсли

$$
-\ln \varphi(t)=o(\omega(t)) \quad \text { nри } t \rightarrow \infty
$$

mo

$$
-\ln \varphi_{S}(t)=(1+o(1)) J(t)+o(\omega(t)) \quad \text { npu } t \rightarrow \infty .
$$

(2) Ecли

$$
-\ln \varphi(t) \sim \omega(t) \quad \text { npu } t \rightarrow \infty
$$

$u$

$$
k_{\tau}=\int_{1}^{\infty} u^{-\tau} d b(u)<\infty
$$

mo

$$
-\ln \varphi_{S}(t) \sim J(t)+\left(\Lambda_{\tau}-k_{\tau}\right) \omega(t) \quad \text { npu } t \rightarrow \infty
$$

гдe

$$
\Lambda_{\tau}=\sum_{j \geqslant 1} \lambda_{j}^{\tau}<\infty .
$$

В [6] также показано, что условия (5.2)-(5.4) являются в некотором смысле необходимыми.

Отметим, что соотношения (5.3) и (5.4) равносильны и заведомо выполняются, если $\tau>\gamma$. 
Лемма 3. Пусть $0 \leqslant \gamma \leqslant 1$ и выполняются условия (2.1), (2.2). Тогда

$$
\liminf _{t \rightarrow \infty} \frac{J(t)}{b(t)} \geqslant K(\gamma)
$$

Кроме того, если $K(\gamma)=\infty$, то

$$
\lim _{t \rightarrow \infty} \frac{-\ln \varphi_{S}(t)}{b(t)}=\infty
$$

u, при $0 \leqslant \gamma<1$,

$$
\lim _{r \rightarrow 0} \frac{-\ln \mathbf{P}(S<r)}{b(s)}=\infty,
$$

где $s=s(r) \rightarrow \infty$ и выполнено условие $b(s) / s \sim r$ при $r \rightarrow 0$.

Д о к а з а т е ль с т в о л е м мы 3 . С помощью (2.2) можно показать, что

$$
J(t)=\int_{1 / t}^{\infty}(b(u t)-b(1)) d \ln \varphi\left(\frac{1}{u}\right) .
$$

Таким образом, при $t \rightarrow \infty$

$$
\begin{aligned}
J(t) & \geqslant \int_{\varepsilon}^{1 / \varepsilon}(b(u t)-b(1)) d \ln \varphi\left(\frac{1}{u}\right) \sim b(t) \int_{\varepsilon}^{1 / \varepsilon} u^{\gamma} d \ln \varphi\left(\frac{1}{u}\right) \\
& =-b(t) \int_{\varepsilon}^{1 / \varepsilon} u^{-\gamma} d \ln \varphi(u),
\end{aligned}
$$

если $\varepsilon$ стремится к 0 достаточно медленно. Отсюда следует (5.5).

Далее, пусть $R \rightarrow \infty$ и $\varepsilon \rightarrow 0$ при $t \rightarrow \infty$ таким образом, что $b_{0}(\varepsilon t) \sim b_{0}(t) \sim b_{0}(t / \varepsilon)$ и $\varepsilon \geqslant\left(t \lambda_{2 R}\right)^{-1}$ (см. обозначения в $\left.(2.1)\right)$.

Обозначим $\lambda(y), y>0$, положительную и неубывающую функцию такую, что $\lambda_{j}=\lambda(j), j \geqslant 1$. Тогда по $(2.1)$ и $[6,(3.1)$ и $(3.2)]$

$$
\begin{aligned}
-\ln \varphi_{S}(t) & \geqslant \sum_{j \geqslant R}\left(-\ln \varphi\left(\lambda_{j} t\right)\right) \geqslant-\int_{R}^{\infty} \ln \varphi(\lambda(y) t) d y \\
& =\int_{R}^{\infty}(y-R) d \ln \varphi(\lambda(y) t) \geqslant 0.5 \int_{2 R}^{\infty} y d \ln \varphi(\lambda(y) t) \\
& \sim 0.5 \int_{2 R}^{\infty} b\left(\frac{1}{\lambda(y)}\right) d \ln \varphi(\lambda(y) t) \geqslant 0.5 \int_{\varepsilon}^{1 / \varepsilon} b(u t) d \ln \varphi\left(\frac{1}{u}\right) \\
& \sim 0.5 b(t) \int_{\varepsilon}^{1 / \varepsilon} u^{\gamma} d \ln \varphi\left(\frac{1}{u}\right) .
\end{aligned}
$$

Последний интеграл стремится к $\infty$, что приводит к (5.6).

Проверка соотношения (5.7) осуществляется при помощи (5.6) так же, как в [5, предложение 1]. Лемма 3 полностью доказана. 
Лемма 4. Пусть выполняются условия (2.8)-(2.12). Тогда

$$
\limsup _{t \rightarrow \infty} \frac{J(t)}{b(t)} \leqslant K(\gamma)<\infty
$$

Д ок аза тельств о ле м мы 4. Случай $\gamma=0$ был рассмотрен в $[5$, лемма 1$]$.

Пусть $0<\gamma<1$. Из условий $(2.8)-(2.12)$ и леммы 1 вытекает, что $K(\gamma)<\infty$. Далее,

$$
J(t)=\left(\int_{1 / \varepsilon}^{\infty}+\int_{\varepsilon}^{1 / \varepsilon}+\int_{1 / t}^{\varepsilon}\right)(b(u t)-b(1)) d \ln \varphi\left(\frac{1}{u}\right)=J_{1}(t)+J_{2}(t)+J_{3}(t) .
$$

Если $\varepsilon$ стремится к 0 при $t \rightarrow \infty$ достаточно медленно, то

$$
J_{2}(t) \sim \int_{\varepsilon}^{1 / \varepsilon} b(u t) d \ln \varphi\left(\frac{1}{u}\right) \sim b(t) \int_{\varepsilon}^{1 / \varepsilon} u^{\gamma} d \ln \varphi\left(\frac{1}{u}\right) \sim K(\gamma) b(t) .
$$

Теперь, используя свойства медленно меняющихся функций (см. [7]) и рассуждая как в [5, доказательство леммы 1$]$, получим с учетом (2.8) (и $\left.\mathbf{E} X^{\gamma}<\infty\right)$ при $t \rightarrow \infty$

$$
J_{1}(t)=O(1)\left(-b(t) \varepsilon^{-\gamma} \ln \varphi(\varepsilon)-\int_{1 / \varepsilon}^{\infty} \ln \varphi\left(\frac{1}{u}\right) b(u t) \frac{d u}{u}\right)=o(b(t)) .
$$

Пусть имеет место (2.9). Тогда $J_{3}(t) \leqslant-b(t) \int_{1 / \varepsilon}^{\infty} h(u) d \ln \varphi(u)=o(b(t))$, $t \rightarrow \infty$. Отсюда и из (5.9)-(5.11) вытекает (5.8).

Пусть теперь выполняются условия $(2.10)-(2.12)$. Положим $J_{4}(t)=$ $-\int_{1 / \varepsilon}^{\varepsilon t} \ln \varphi(u) b(t / u) / u d u$. Из (2.11) следует, что

$$
J_{3}(t)=O\left(J_{4}(t)\right)+o(b(t)) \quad \text { при } t \rightarrow \infty,
$$

если $\varepsilon$ стремится к 0 достаточно медленно.

Далее,

$$
J_{4}(t)=-\left(\int_{1 / \varepsilon}^{\sqrt{t}}+\int_{\sqrt{t}}^{\varepsilon t}\right) \ln \varphi(u) b\left(\frac{t}{u}\right) \frac{d u}{u}=I_{1}(t)+I_{2}(t),
$$

где

$$
I_{1}(t) \leqslant-b(t) \int_{1 / \varepsilon}^{\sqrt{t}} \ln \varphi(u) \hat{h}(u) \frac{d u}{u}, \quad \hat{h}(u)=\sup _{t \geqslant u^{2}} \frac{b(t / u)}{b(t)} .
$$

Имея в виду $(2.11),(2.12)$ и равенство $\hat{h}(u)=\tilde{h}(u)($ см. $(2.3))$, получим

$$
I_{1}(t)=o(b(t)) \quad \text { при } t \rightarrow \infty .
$$


Имеем

$$
\begin{aligned}
\frac{I_{2}(t)}{b(t)} & =O(1) \int_{\sqrt{t}}^{\varepsilon t} \kappa(u) b(u) \frac{b(t / u)}{b(t)} \frac{d u}{u}=O(1) \int_{1 / \varepsilon}^{\sqrt{t}} \kappa(s) b(s) \frac{b(t / s)}{b(t)} \frac{\kappa(t / s)}{\kappa(s)} \frac{d s}{s} \\
& =O(1) \int_{1 / \varepsilon}^{\sqrt{t}} \kappa(s) b(s) \hat{h}(s) \frac{\kappa(t / s)}{\kappa(s)} \frac{d s}{s}
\end{aligned}
$$

Следовательно, по (2.10) и (2.12)

$$
I_{2}(t)=O(b(t)) \int_{1 / \varepsilon}^{\infty} \kappa(s) b(s) \tilde{h}(s) \frac{d s}{s}=o(b(t)) \quad \text { при } t \rightarrow \infty .
$$

Отсюда и из (5.9)-(5.14) снова следует (5.8).

Лемма 4, таким образом, полностью доказана.

Д о к а з а т е л с с т о т е о р е м ы 4. Первое утверждение вытекает из леммы $2(1)$ и лемм 3 (неравенство (5.5)) и 4; второе утверждение является следствием леммы 3 (соотношение (5.6)).

Д ок а з а тель с тв а след с т в й й 1,2 и 3 базируются, в основном, на теореме 4 и замечании 2. Проверим для примера достаточность в следствии 1 при $0<A=C(\gamma)<\infty$ и $b(u)=u^{\gamma} L(u)$, когда $0<\gamma<1$. Действительно, первое предположение дает $K(\gamma)<\infty$ и, следовательно, $-\ln \varphi(t)=o\left(t^{\gamma}\right), t \rightarrow \infty$, а второе ведет к $h(u)=O\left(u^{-\gamma}\right)$, $u \rightarrow \infty$, и к (2.16). Значит, выполняются условия (2.8) и (2.9). Остается воспользоваться теоремой 4.

Все остальные рассуждения проводятся аналогично.

Д о к а з а т е л с т в о т е о р е м ы 5 легко следует из первого утверждения леммы 2 и (3.1). Так, если $\gamma>0$, то, полагая $b(u)=u^{\gamma}$, получим $J(t)=\gamma t^{\gamma} l_{\gamma}(t)+O(1) \sim \gamma t^{\gamma} l_{\gamma}(t), t \rightarrow \infty$.

У тверждение о том, что (3.1) равносильно медленному изменению на бесконечности интеграла $l_{\gamma}(t)$, можно проверить, исходя из определения, с помощью оценок

$$
\begin{aligned}
& 1 \leqslant \frac{l_{\gamma}(k t)}{l_{\gamma}(t)} \leqslant 1+(-\ln \varphi(k t)) \int_{t}^{k t} u^{-1-\gamma} d u \\
& \frac{l_{\gamma}(k t)}{l_{\gamma}(t)} \geqslant 1+(-\ln \varphi(t)) \int_{t}^{k t} u^{-1-\gamma} d u
\end{aligned}
$$

при $k>1$ (и аналогичных соотношений при $0<k<1$ ).

Д оказ а тельст в о т е оремы 6 . При любом $\varepsilon \in(0,1)$ имеем

$$
J(t)=\left(\int_{1}^{\varepsilon t}+\int_{\varepsilon t}^{t / \varepsilon}+\int_{t / \varepsilon}^{\infty}\right)\left(-\ln \varphi\left(\frac{t}{u}\right)\right) d b_{\gamma}(u)=I_{1}+I_{2}+I_{3} .
$$

Функция $l_{\gamma}(t)$ медленно меняется на $\infty$ и $\lim _{t \rightarrow \infty} t^{\gamma} l_{\gamma}(t) / b(t)=\infty$; кроме того, если $\gamma=0$, то $\lim _{t \rightarrow \infty} l_{\gamma}(t) / \ln t=\infty$. 
Значит, если $\varepsilon$ стремится к 0 достаточно медленно при $t \rightarrow \infty$, то $I_{1} \sim c(\gamma) l_{\gamma}(\varepsilon t) \sim c(\gamma) l_{\gamma}(t)$ и $I_{2}=o\left(t^{\gamma} l_{\gamma}(t)\right)$ при $t \rightarrow \infty$. Действительно, при положительных $\gamma$, обозначив $u^{-\gamma} b_{\gamma}(u)$ через $L(u)$, получим

$$
I_{2} \leqslant\left(-\ln \varphi\left(\frac{1}{\varepsilon}\right)\right) t^{\gamma}\left(\varepsilon^{-\gamma} L\left(\frac{t}{\varepsilon}\right)-\varepsilon^{\gamma} L(\varepsilon t)\right)=O(1) \varepsilon^{-2 \gamma} b_{\gamma}(t)=o\left(t^{\gamma} l_{\gamma}(t)\right) .
$$

Применяя [5, рассуждения после $(27)]$, мы также найдем $I_{3}=o(b(t))=$ $o\left(t^{\gamma} l_{\gamma}(t)\right)$ при $t \rightarrow \infty$.

Случай $\gamma=0$ изучается схожим образом. Теперь, чтобы доказать теорему 6 , достаточно воспользоваться леммой $2(1)$.

Д ок аз а тель с тв о т е о ре мы 7. Если имеет место (3.8), то соотношение $l_{\gamma}(1)<\infty$ равносильно (2.15). Далее, в представлении $(5.15)$

$$
I_{3} \sim c t^{\gamma} \int_{t / \varepsilon}^{\infty} u^{-\gamma} d b_{\gamma}(u) \sim c \gamma t^{\gamma} \int_{t / \varepsilon}^{\infty} b_{\gamma}(u) u^{-1-\gamma} d u
$$

и $\left(\right.$ см. (5.16)) $I_{2}+I_{3} \sim c \gamma t^{\gamma} l_{\gamma}(t), t \rightarrow \infty$. В силу (3.9)

$$
I_{1}=O\left(t^{\delta}\right) \int_{1}^{\varepsilon t} u^{-\delta} d b_{\gamma}(u)=O(1) \varepsilon^{\gamma-\delta} b_{\gamma}(t)=o(1) t^{\gamma} l_{\gamma}(t), \quad t \rightarrow \infty .
$$

Следовательно, $J(t) \sim c \gamma t^{\gamma} l_{\gamma}(t), t \rightarrow \infty$. Кроме того, из (3.9) следует (5.1). Теперь, чтобы завершить доказательство, следует применить лемму $2(1)$.

Д ок азательст в о з а м е ч а н и я 6 . Оценим заново интеграл $I_{1}$ из предыдущих рассуждений с учетом новых предположений. Из (3.1) следует, что $I_{1}=t^{\gamma}\left(I_{11}+I_{12}+o(1) l_{\gamma}(t)\right), t \rightarrow \infty$, где $I_{11}=-\int_{1 / \varepsilon}^{\sqrt{t}} t^{-\gamma} \ln \varphi(t / u) d b_{\gamma}(u)$ и $I_{12}=-\int_{\sqrt{t}}^{\varepsilon t} t^{-\gamma} \ln \varphi(t / u) d b_{\gamma}(u)$. Имеем: $I_{11}=o(1) \int_{1 / \varepsilon}^{\sqrt{t}} l_{\gamma}(t / u) L(u) d u / u$ и

$$
I_{12}=o(1)\left(-\int_{\sqrt{t}}^{\varepsilon t} l_{\gamma}\left(\frac{t}{u}\right) d l_{\gamma}(u)\right)=o(1)\left(l_{\gamma}^{2}(\sqrt{t})+\int_{1 / \varepsilon}^{\sqrt{t}} l_{\gamma}\left(\frac{t}{u}\right) L(u) \frac{d u}{u}\right) .
$$

С учетом (3.10) и оценок $\int_{\sqrt{t}}^{\varepsilon t} l_{\gamma}(t / u) L(u) d u / u \leqslant l_{\gamma}(t) \int_{1 / \varepsilon}^{\infty} \tilde{l}_{\gamma}(u) L(u) d u / u$ и $l_{\gamma}^{2}(t) \leqslant 2 l_{\gamma}(\sqrt{t}) \int_{\sqrt{t}}^{\infty} l_{\gamma}(t / u) L(u) d u / u$, отсюда получим соотношение $I_{1}=$ $o\left(t^{\gamma} l_{\gamma}(t)\right), t \rightarrow \infty$ и, как следствие, замечание $6(1)$.

Проверим второе утверждение. С помощью (3.12) найдем

$$
\begin{aligned}
I_{11} & =O(1) \int_{1 / \varepsilon}^{\sqrt{t}} \kappa\left(\frac{t}{u}\right) l_{\gamma}\left(\frac{t}{u}\right) L(u) \frac{d u}{u}=O(1) l_{\gamma}(t) \int_{1 / \varepsilon}^{\sqrt{t}} \kappa(u) \tilde{l}_{\gamma}(u) L(u) \frac{d u}{u} \\
I_{12} & =O(1) \int_{1 / \varepsilon}^{\sqrt{t}} \kappa(u) l_{\gamma}(u) L\left(\frac{t}{u}\right) \frac{d u}{u}=O(1) \int_{1 / \varepsilon}^{\sqrt{t}} \frac{\mu(t / u)}{\mu(u)} l_{\gamma}\left(\frac{t}{u}\right) \kappa(u) L(u) \frac{d u}{u} \\
& =O(1) l_{\gamma}(t) \int_{1 / \varepsilon}^{\infty} \bar{\mu}(u) \kappa(u) \tilde{l}_{\gamma}(u) L(u) \frac{d u}{u} .
\end{aligned}
$$


Отсюда и из (3.11) вновь следует нужная оценка для $I_{1}$. Таким образом, замечание 6 полностью доказано.

Д ок аз а те ль с т в о т е о ре м 8-10. Пусть положительное $\varepsilon=\varepsilon(t)$ стремится к 0 достаточно медленно при $t \rightarrow \infty$. Аналогично (5.15), запишем

$$
\begin{aligned}
J(t) & =\left(\int_{1}^{1 / \varepsilon}+\int_{1 / \varepsilon}^{\sqrt{t}}+\int_{\sqrt{t}}^{\varepsilon t}+\int_{\varepsilon t}^{t / \varepsilon}+\int_{t / \varepsilon}^{\infty}\right)\left(-\ln \varphi\left(\frac{t}{u}\right)\right) d b_{\gamma}(u) \\
& =J_{1}+\cdots+J_{5} .
\end{aligned}
$$

Если $\delta>\gamma$, то в соответствии со свойствами медленно меняющихся функций (см., например, [7]) $\int_{1}^{\infty} u^{-\delta^{\prime}} d b(u)<\infty$ при некотором $\delta^{\prime}, \gamma<$ $\delta^{\prime}<\delta$, а также $k_{\delta}<\infty$ (см. (5.3)) и

$$
J_{1} \sim k_{\delta} \omega_{\delta}(t) \text { при } t \rightarrow \infty \text {. }
$$

Кроме того, при $t \rightarrow \infty$

$$
\begin{aligned}
J_{2}+J_{3} & =O(1) \omega_{\delta}(t) \int_{1 / \varepsilon}^{\infty} u^{-\delta^{\prime}} d b(u)=o\left(\omega_{\delta}(t)\right), \\
J_{4} & =O(1) b_{\gamma}(t)\left(-\ln \varphi\left(\frac{1}{\varepsilon}\right)\right)=o\left(\omega_{\delta}(t)\right) .
\end{aligned}
$$

Используя в $[5$, рассуждения после $(2.7)]$ оценку $b(t u) \leqslant g(t) b(u)$ (см. $(2.3)$ и лемму $1(1))$, получим

$$
J_{5}=o\left(g_{\gamma}(t)\right)=o\left(\omega_{\delta}(t)\right) .
$$

Проделанные выкладки приводят к соотношению

$$
J(t) \sim k_{\delta} \omega_{\delta}(t), \quad t \rightarrow \infty .
$$

Теорема 8 следует из (5.22) и леммы $2(2)$.

Проверим теорему 9. Из (4.5) вытекают соотношения (5.3), (5.4) при $\tau=\gamma$ и, следовательно, (5.19), а (4.6) влечет за собой (2.2). Остается воспользоваться леммой $2(2)$, подходящим образом оценив $J(t)$ (вернее, $\left.J_{2}+\cdots+J_{5}\right)$.

С этой целью, применяя, по аналогии с (5.21), в рассуждениях из [5] оценку $b(t u) \leqslant \omega_{\gamma}(t) \bar{\nu}(u) \bar{\omega}(u) u^{\gamma}$ и имея в виду $(4.6)$, получим

$$
J_{5}=o\left(\omega_{\gamma}(t)\right) \quad \text { при } t \rightarrow \infty \text {. }
$$

Далее, с помощью (4.1), (4.5) и вытекающего из (4.5) соотношения

$$
\lim _{u \rightarrow \infty} \bar{\nu}(u) \widetilde{\omega}(u) \omega(u)=0
$$


найдем, что при $t \rightarrow \infty$

$$
\begin{aligned}
J_{2} & =O(1) \int_{1 / \varepsilon}^{\sqrt{t}} \omega_{\gamma}\left(\frac{t}{u}\right) b_{\gamma}(u) \frac{d u}{u}+o\left(\omega_{\gamma}(t)\right) \\
& =O\left(\omega_{\gamma}(t)\right) \int_{1 / \varepsilon}^{\sqrt{t}} \nu(u) \frac{\omega(t / u)}{\omega(t)} \omega(u) \frac{d u}{u}+o\left(\omega_{\gamma}(t)\right)=o\left(\omega_{\gamma}(t)\right)
\end{aligned}
$$

и аналогично (с учетом неравенства $\bar{\nu}(t / u) \leqslant \bar{\nu}(u), \sqrt{t} \leqslant u<t$ )

$$
J_{3}=o\left(\omega_{\gamma}(t)\right)
$$

Если $\bar{\nu}(\infty)=0$, то оценка (5.20) (при $\delta=\gamma$ ) остается справедливой. Отсюда и из (5.19), (5.23)-(5.25) следует (5.22) и, в конечном счете, теорема 9 (1).

Если же $\bar{\nu}(\infty)>0$, то (4.5) и (4.6) приводят к $K(\gamma)<\infty$ и к асимптотике (при $t \rightarrow \infty$ )

$$
\begin{aligned}
J_{4} & \sim \gamma \int_{\varepsilon t}^{t / \varepsilon}\left(-\ln \varphi\left(\frac{t}{u}\right)\right) b_{\gamma}(u) \frac{d u}{u} \sim \gamma b_{\gamma}(t) \int_{\varepsilon}^{1 / \varepsilon}(-\ln \varphi(u)) \frac{d u}{u^{1+\gamma}} \\
& \sim \gamma b_{\gamma}(t) \int_{0}^{\infty}(-\ln \varphi(u)) \frac{d u}{u^{1+\gamma}}=K(\gamma) b_{\gamma}(t) .
\end{aligned}
$$

Таким образом (см. (5.19), (5.23)-(5.25))

$$
J(t) \sim k_{\delta} \omega_{\delta}(t)+K(\gamma) b_{\gamma}(t) \quad \text { при } t \rightarrow \infty .
$$

Подставляя эту оценку в лемму $2(2)$, приходим к теореме $9(2)$.

Теорема 10 проверяется так же, как теорема $9(2)$, с тем отличием, что вместо (5.20) применяется соотношение (5.16), а (5.21) меняется на (5.17).

При проверке замечания 8 используется [5, замечание 2]. Для доказательства утверждения, следующего за замечанием 8 (и аналогичных результатов после леммы 1 и замечания 6), применяется такое рассуждение:

$$
\ln \frac{1}{\omega\left(u e^{t}\right)}=\ln \frac{1}{\omega(u)}+t\left(\ln \frac{1}{\omega\left(u e^{\theta}\right)}\right)^{\prime} u e^{\theta}, \quad 0<\theta<t
$$

При $t=\ln u$ отсюда следует, что $\ln \left(u e^{\theta}\right) \in(\ln u, 2 \ln u)$ и, по (4.9),

$$
\ln \frac{1}{\omega\left(u^{2}\right)}=\ln \frac{1}{\omega(u)}+O(1), \quad u \rightarrow \infty .
$$




\section{6. Приложение.}

Лемма 5 (см. $[8$, следствия 5 и 6], [5, замечание 3$])$. Пусть $\lambda_{\gamma}(t)=$ $t^{\gamma} \lambda(t), t>0$, где $\gamma \in[0,1)$ и положительная функиия $\lambda(t)$ медленно меняется на $\infty$, и пусть положительная функция $s=s(r), r>0$, стремится $\kappa \infty$ и удовлетворяет условию $\lambda_{\gamma}(s) / s \sim r$ при $r \rightarrow 0$. Тогда:

(1) $-\ln \varphi(t) \sim \lambda_{\gamma}(t), t \rightarrow \infty$, тогда и только тогда, когда $-\ln V(r) \sim c_{*}(\gamma) \lambda_{\gamma}(s), r \rightarrow 0$, где $c_{*}(\gamma)=(1-\gamma) \gamma^{\gamma /(1-\gamma)}$;

$(2)-\ln \varphi(t)=O\left(\lambda_{\gamma}(t)\right), t \rightarrow \infty$, тогда и только тогда, когда $-\ln V(r)=O\left(\lambda_{\gamma}(s)\right), r \rightarrow 0$, и аналогично в случае замень $O(\cdots)$ на $o(\cdots)$.

Кроме того, если $\lambda\left(t \lambda^{1 /(1-\gamma)}(t)\right) \sim \lambda(t), t \rightarrow \infty$, то

$$
\lambda_{\gamma}(s) \sim\left(\frac{1}{r}\right)^{\gamma /(1-\gamma)} \lambda^{1 /(1-\gamma)}\left(\left(\frac{1}{r}\right)^{1 /(1-\gamma)}\right) .
$$

\section{СПИСОК ЛИТЕРАТУРЫ}

1. Aurzada $F$. On the lower tail probabilities of some random sequences in $l_{p}$. $-\mathrm{J}$. Theoret. Probab., 2007, v. 20, № 4, p. 843-858.

2. Aurzada F. A short note on small deviations of sequences of i.i.d. random variables with exponentially decreasing weights. - Statist. Probab. Lett., 2008, v. 78, № 15, p. 2300-2307.

3. Borovkov A.A., Ruzankin P.S. On small deviations of series of weighted random variables. - J. Theoret. Probab., 2008, v. 21, № 3, p. 628-649.

4. Боровков А. А., Рузанкин П. С. О малых уклонениях рядов независимых положительных случайных величин с весами, близкими к экспоненциальным. - Матем. тр., 2008, т. 11, № 1, с. 49-67.

5. Rozovsky L. V. Small deviations of series of weighted i.i.d. non-negative random variables with a positive mass at the origin. - Statist. Probab. Lett., 2009, v. 79, № 13, p. 1495-1500.

6. Rozovsky L. V. On the behavior of the $\log$ Laplace transform of series of weighted nonnegative random variables at infinity. - Statist. Probab. Lett., 2010, v. 80, № 9-10, p. $764-770$.

7. Bingham N. H., Goldie C. M., Teugels J. L. Regular Variation. Cambridge: Cambridge Univ. Press, 1987, $491 \mathrm{p}$.

8. Rozovsky L. V. Remarks on a link between Laplace transform and distribution function of a nonnegative random variable. - Statist. Probab. Lett., 2009, v. 79, № 13, p. 1501-1508.

9. Розовский Л. В. О малых уклонениях сумм взвешенных положительных случайных величин. - Зап. науч. сем. ПОМИ, 2010, т. 384, с. 212-224.

Поступила в редакцию

10.II.2010

Исправленный вариант 11.IV.2011 